\title{
Two-Dimensional Dispersive Off-Chip Beam Scanner Fabricated on Silicon-On-Insulator
}

\author{
Karel Van Acoleyen, Student Member, IEEE, Wim Bogaerts, Member, IEEE, and Roel Baets, Fellow, IEEE
}

\begin{abstract}
One of the key elements in optical wireless applications such as laser scanning and writing is beam steering. While beam steering can be done actively using mirrors or liquid crystals, we have taken a passive approach to steer a beam in two dimensions by wavelength tuning. Therefore, we have fabricated an integrated two-dimensional beam steerer using silicon-on-insulator. The beam width is around $4.0^{\circ}$ and can be steered in a range of $15^{\circ} \times 50^{\circ}$ for a wavelength shift of $100 \mathrm{~nm}$. The beam width and range can be further optimized by changing the grating structure and size of the component.
\end{abstract}

Index Terms - Beam steering, demultiplexing, grating couplers, silicon-on-insulator (SOI).

\section{INTRODUCTION}

$\mathbf{O}$ PTICAL beam steering is of key importance in free-space optical applications. Several beam steering methods have been developed such as MEMS (MicroElectroMechanical systems), movable mirrors, acousto-optic deflectors or optical phased arrays (OPAs) [1]. An OPA consists of an array of radiating apertures of which the phase can be controlled. In [2], such an integrated OPA on silicon-on-insulator (SOI) has been demonstrated using thermo-optic phase tuning. While these approaches need active steering of all the elements, beam steering can also be done by wavelength tuning. When using diffractive elements, the direction of emission can be controlled by wavelength tuning. A two-dimensional space can then be scanned by using two dispersive elements as has been shown in [3].

In this letter, we present a fully integrated approach to perform two-dimensional off-chip wavelength scanning using the silicon photonics platform. The steering principle is based on scanning a beam slowly in one direction while it is steered very fast in the other direction using a low and high order grating, respectively. The high order grating consists of an Arrayed Waveguide Grating (AWG). The low order grating is the out-coupling grating which performs two tasks: coupling the light off-chip and steering the beam slowly in one direction. The steering speed is determined by the wavelength sweeping speed, which

Manuscript received April 08, 2011; revised June 03, 2011; accepted June 08, 2011. Date of publication June 16, 2011; date of current version August 17, 2011. The work of K. Van Acoleyen and W. Bogaerts was supported by the Research Foundation-Flanders (FWO) under a research grant and a postdoctoral fellowship, respectively. This work was supported by the Methusalem project "Smart Photonic ICs" of Ghent University and by the IAP project Photonics@be.

The authors are with the Photonics Research Group, Department of Information Technology (INTEC), Ghent University-IMEC, 9000 Ghent, Belgium (e-mail: karel.vanacoleyen@intec.ugent.be; wim.bogaerts@intec.ugent.be; roel.baets@intec.ugent.be).

Color versions of one or more of the figures in this letter are available online at http://ieeexplore.ieee.org.

Digital Object Identifier 10.1109/LPT.2011.2159785

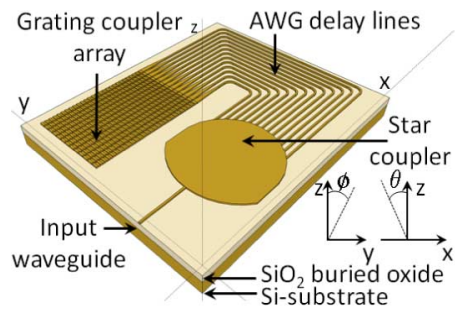

(a)

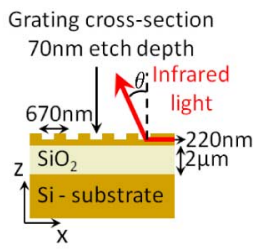

(b)
Fig. 1. (a) Two-dimensional beam scanner on SOI. (b) Cross-section of the grating coupler array.

can happen very fast. The basic principles and results of this component have been shown in [4], while in this Letter we give a more in debt analysis of the power efficiency, measured wavelength range and optimization possibilities.

These beam steering elements can find applications in laser scanning, spectroscopy, demultiplexing [5] and spectral pulse shaping applications [6]. Next, we discuss the design and fabrication of the component. Section III will give the measurement results. Finally, the conclusion is presented in Section IV.

\section{DESIGN AND FABRICATION}

The component shown in Fig. 1 was fabricated on SOI at imec, using standard CMOS (Complementary Metal Oxide Semiconductor) processes [7]. The SOI wafer has a $2 \mu \mathrm{m}$ buried oxide layer and a $220 \mathrm{~nm}$ silicon top layer. An etch of $220 \mathrm{~nm}$ was used to etch the waveguides and the star coupler and an etch of $70 \mathrm{~nm}$ to define the grating couplers and tapering sections of the star coupler. Light is guided from an optical fiber into the structure using a grating coupler for near vertical coupling of the TE (Transverse Electric)-like mode [8]. The waveguide is then adiabatically tapered to a $450 \mathrm{~nm}$ wide photonic wire which is the input waveguide shown in Fig. 1(a). Light is then split through a star coupler into 16 waveguides which are spaced at a distance of $2 \mu \mathrm{m}$. The delay lines have a width of $800 \mathrm{~nm}$ which reduces phase errors. There is a fixed delay length $\Delta L$ between each waveguide, which forms the AWG. At the end of each waveguide, the photonic wire tapers to a $800 \mathrm{~nm}$ wide wire on which a grating is etched. The period of the grating is $670 \mathrm{~nm}$ with a fill factor of $50 \%$ (Fig. 1(b)).

The grating coupler consists of a second order grating and will perform two functions: coupling light out-of-plane and steering the light slowly in the $\theta$-direction. The steering in the $\theta$-direction (Fig. 1) is given by the grating equation:

$$
\sin \theta=\frac{\Lambda_{g r} n_{e f f, g r}-\lambda}{n_{c t} \Lambda_{g r}},
$$

with $\Lambda_{g r}$ the period of the grating $\left(\Lambda_{g r}=670 \mathrm{~nm}\right), \lambda$ the free-space wavelength, $n_{\text {eff.gr }}$ the effective index of the guided 
mode in the grating area and $n_{c t}$ the refractive index of the background which is air $\left(n_{c t}=1\right)$ in this case. The steering speed is given by

$$
\frac{d \theta}{d \lambda} \approx \frac{d \sin \theta}{d \lambda}=\frac{d n_{e f f, g r}}{d \lambda}-\frac{1}{\Lambda_{g r}}
$$

where the approximation is valid when the angle $\theta$ is relatively small. There is also an important dispersion factor $d n_{e f f, g r} / d \lambda$. Whereas material dispersion is relatively weak for the wavelength range considered here, the waveguide dispersion has a significant influence due to the extremely high confinement in the small photonic wires. This dispersion factor is about $-8.9 \times$ $10^{-4} / \mathrm{nm}$ for a $70 \mathrm{~nm}$ deep etched grating in a $800 \mathrm{~nm}$ wide waveguide.

While the beam is scanned slowly in the $\theta$-direction, a fast steering is performed in the $\phi$-direction (Fig. 1(a)) due to the AWG. The grating couplers are placed in a $N$-array configuration with positions $\mathbf{s}_{n}=n \Lambda_{y} \mathbf{u}_{y}$, with $\Lambda_{y}$ the spacing of the elements in the $y$-direction and $n=0 \ldots N-1$. The far field can be calculated by multiplication of the far field of one grating coupler with the array factor $T$ :

$$
\begin{aligned}
T & =\sum_{n=0}^{N-1} A_{n} e^{-j \beta_{n}} e^{j \mathbf{k} \cdot \mathbf{s}_{n}} \\
& =\sum_{n=0}^{N-1} A_{n} e^{-j \beta_{n}} e^{j k_{0}\left[n \Lambda_{y} \sin \phi\right]},
\end{aligned}
$$

where $\beta_{n}$ is the phase delay between the elements, $\mathbf{k}$ is the wave vector (in air) with magnitude $k_{0}$ and $A_{n}$ is the amplitude of each element which is further assumed to be 1 . The length difference $\Delta L$ results in a phase delay of $\beta_{n}=n_{e f f}(2 \pi / \lambda) n \Delta L$, with $n_{\text {eff }}$ the effective index of the fundamental TE-like mode. The array factor can then be calculated in closed form:

$$
\begin{aligned}
& T=e^{j\left[\left(k_{0} \Lambda_{y} \sin \phi-k_{0} n_{e f f} \Delta L\right)(N-1) / 2\right]} \\
& \times \frac{\sin \left(N \frac{k_{0} \Lambda_{y} \sin \phi-k_{0} n_{e f f} \Delta L}{2}\right)}{\sin \left(\frac{k_{0} \Lambda_{y} \sin \phi-k_{0} n_{e f f} \Delta L}{2}\right)} .
\end{aligned}
$$

The array factor is maximum in the $\phi$-direction for

$$
\sin \phi=q \frac{\lambda}{\Lambda_{y}}+\frac{n_{e f f} \Delta L}{\Lambda_{y}},
$$

with $q$ an integer. Due to the delay lines, the absolute value of $q$ will be large. The beams will shift at a rate of

$$
\frac{d \phi}{d \lambda} \approx \frac{d \sin \phi}{d \lambda}=\frac{q}{\Lambda_{y}}+\frac{d n_{e f f}}{d \lambda} \frac{\Delta L}{\Lambda_{y}},
$$

where the angle $\phi$ is assumed to be relatively small. Note that $q$ will be negative so that the beam will shift in the negative $\phi$-direction for increasing wavelength. The effective index at $\lambda=1550 \mathrm{~nm}$ for this wire is $n_{\text {eff }} \approx 2.65$. The change of $n_{\text {eff }}$ with wavelength for a $800 \mathrm{~nm}$ wide wire is negative as well and is about $-8.2 \times 10^{-4} / \mathrm{nm}$ around $\lambda=1550 \mathrm{~nm}$. The waveguide dispersion is smaller in a wider waveguide. For comparison, the dispersion value is about $-0.013 / \mathrm{nm}$ in a $450 \mathrm{~nm}$ wide wire. However, the factor $\Delta L / \Lambda_{y}$ can become large so that the influence of dispersion cannot be neglected.

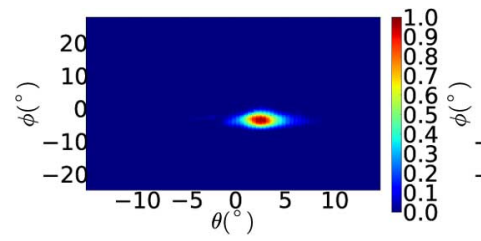

(a)

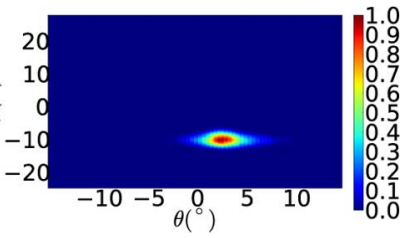

(b)
Fig. 2. Far-field pattern of a beam steering component with an AWG of order 150 at different wavelengths: (a) $1549 \mathrm{~nm}$, (b) $1550 \mathrm{~nm}$.

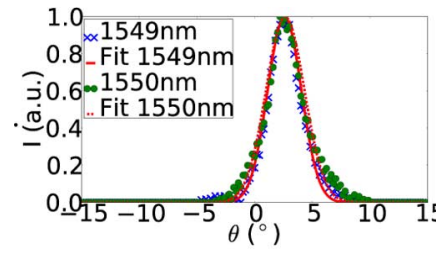

(a)

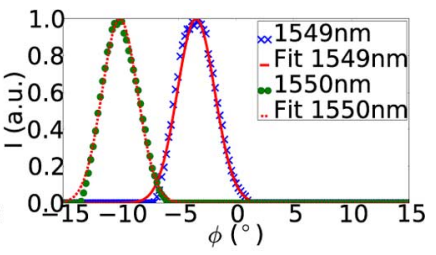

(b)
Fig. 3. Cross-sectional plots of a beam steerer with an AWG of order 150 at a wavelength of 1549 and $1550 \mathrm{~nm}$. (a) Along $\theta$, the shift is smaller than the measurement capabilities. (b) Along $\phi$, a shift of approximately $6.5^{\circ}$ can be seen.

\section{MEASuREMEnt Results AND Discussion}

A Fourier imaging setup was used to investigate the far field pattern of the components [9]. The far field is imaged onto the back-focal plane of a microscope objective (MO). In this plane, one point corresponds to a specific direction of emission. This plane is then brought back to the infrared camera by means of two lenses. The numerical aperture (NA) of the microscope objective determines the maximum direction of emission that is captured by the measurement setup. An MO with a NA of 0.5 was used meaning that the maximum angle with respect to the normal of the surface that can be measured is $30^{\circ}$.

Different wavelength scanners were fabricated and measured for a wavelength range of $100 \mathrm{~nm}$. The only difference between the components is the order $q$ of the AWG, which determines the steering speed in the $\phi$-direction given by (6). Fig. 2 shows the far field of a beam steering component with an AWG of order 150 at different wavelengths. While the beam width in the $\theta$-direction depends on the out-coupling strength of the grating which is wavelength dependent (Fig. 5(a)), the main reason why the beam profile becomes wider at wavelengths where $\phi$ is large, is due to aberrations in the Fourier imaging setup for the extreme off-axis case. In Fig. 3 a cross-sectional plot of the measured far fields is given. The mean out-coupling angle is measured to be $\theta_{0}=2.9^{\circ}$ at $\lambda=1550 \mathrm{~nm}$. The full-width-half-maximum (FWHM) beam width is $3.5^{\circ}-4.0^{\circ}$ in both the $\theta$ - and $\phi$-direction. We can clearly see a very small shift in the $\theta$-direction (smaller than the measurement capabilities), while the beam shifts about $6.5^{\circ}$ in the $\phi$-direction. The position of the beam for one of the fabricated components with an AWG of order 150 can be found in Fig. 4. The $\theta$-angle varies slowly while the $\phi$-angle varies quickly when changing the wavelength. At each jump, we focus on a different lobe emitted by the grating array. This lobe then shifts out of the measurement range until a new lobe appears. The steering speed given by (2) is measured to be $d \theta / d \lambda \approx 0.148^{\circ} / \mathrm{nm}$. The steering speed in the $\phi$-direction given by (6) depends mainly on the order of the AWG and thus on the length difference $\Delta L$. The results are summarized in 


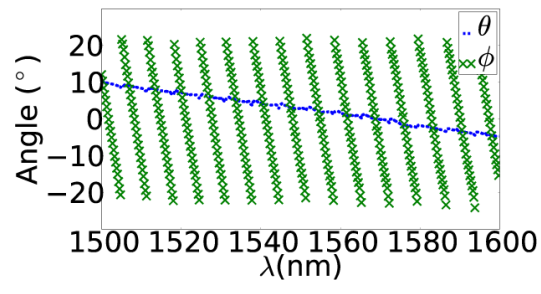

Fig. 4. $\theta$ and $\phi$ position of the beam of a beam steerer with an AWG of order 150 as a function of wavelength.

TABLE I

Theoretical and Measured Results of the Beam SteErers. THe Last Column Gives the AWG Free Spectral Range (FSR)

\begin{tabular}{ccccc}
$\Delta L(\mu \mathrm{m})$ & $\mathrm{q}$ & $\begin{array}{c}\mathrm{d} \phi / \mathrm{d} \lambda\left({ }^{\circ} / \mathrm{nm}\right) \\
\text { (theory) }\end{array}$ & $\begin{array}{c}\mathrm{d} \phi / \mathrm{d} \lambda\left({ }^{\circ} / \mathrm{nm}\right) \\
\text { (meas.) }\end{array}$ & FSR $(\mathrm{nm})$ \\
\hline 29.2 & -50 & -2.1 & -2.2 & 22.7 \\
58.5 & -100 & -4.2 & -4.4 & 11.4 \\
87.7 & -150 & -6.4 & -6.5 & 7.7 \\
117.0 & -200 & -8.5 & -8.7 & 5.7 \\
146.2 & -250 & -10.6 & -10.7 & 4.7 \\
\hline
\end{tabular}

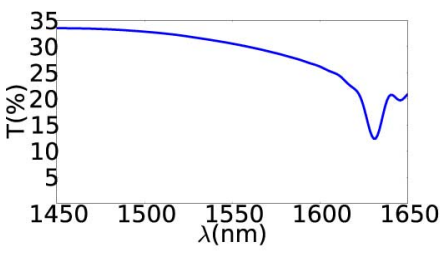

(a)

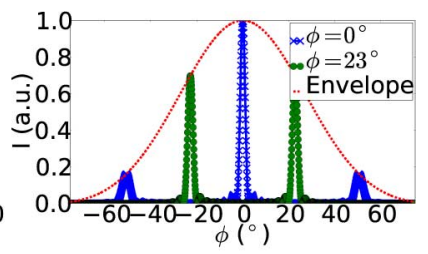

(b)
Fig. 5. (a) Simulated out-coupling efficiency of one grating; the drop corresponds to the second-order reflection of the grating. (b) Simulated spectrum of the wavelength scanner for the two extreme cases of steering in the $\phi$-direction: $\phi=0^{\circ}$ and $\phi=23^{\circ}$. The red dashed line shows the envelope of the far field corresponding to the far field of one grating coupler.

Table I for the fabricated components. A good agreement with the theoretical expected values can be seen.

Fig. 5(a) shows the out-coupling efficiency calculated using a 3-D-FDTD (Finite Difference Time Domain) simulation. The efficiency varies from $33 \%$ at $1500 \mathrm{~nm}$ to $26 \%$ at $1600 \mathrm{~nm}$. The dip near $1630 \mathrm{~nm}$ corresponds to the wavelength where the grating acts as a second order reflection grating. By changing the period of the grating, we can shift the field-of-view to another angular range to ensure operation where the grating strongly couples out light. The efficiency of coupling light off-chip can be greatly increased using an extra silicon overlay as shown in [8]. In Fig. 5(b), we can see the simulated angular spectrum for the two extreme cases of steering in the $\phi$-direction. For wavelengths where $\phi=0^{\circ}$, about $40 \%$ is emitted in the main lobe and $25 \%$ is emitted through the two other orders of emission, while the remaining $10 \%$ is lost in sidelobes between the different output orders. For wavelengths where $\phi=23^{\circ}$, we are at the situation where a jump occurs to another output order (Fig. 4). Both output orders now have an equal power of about $45 \%$. In this case, both output orders (and thus almost all the light emitted) can be captured by the NA of the MO and the efficiency from input waveguide to free-space was measured to be roughly $10-13 \%$ around $1550 \mathrm{~nm}$. The discrepancy with Fig. 5(a) is mainly due to the loss in the star coupler which is about $1.5 \mathrm{~dB}$ and the waveguide loss which is approximately $1 \mathrm{~dB}$. The $3 \mathrm{~dB}$ field-of-view in the $\phi$-direction of the scanner is determined by the FWHM of the far field of one grating cou- pler. This is shown as the red dashed line in Fig. 5(b) where the FWHM is $63^{\circ}$.

In order to scan the complete 2-D space, the beam should shift about $50^{\circ}$ in the $\phi$-direction when it has shifted $4^{\circ}$ in the $\theta$-direction (as the FWHM beam width is around $4^{\circ}$ ). The latter happens over a wavelength range of about $27 \mathrm{~nm}$. The minimum steering speed in the $\phi$-direction should thus be around $1.85^{\circ} / \mathrm{nm}$. This results in a wavelength resolution of around $2 \mathrm{~nm}$ over a $100 \mathrm{~nm}$ wavelength range, or 50 resolvable spots in the 2-D angular space. The main limitation on this performance is due to the beam width.

The beam width can be decreased in the $\phi$-direction by having more waveguides. However, when the delay lines become too long, phase errors due to sidewall roughness will broaden the beam, which was already the case for the higher order AWG $(q=250)$. This can be overcome by optimizing the fabrication method to reduce the sidewall roughness of the delay lines. The beam width in the $\theta$-direction depends on the strength of the grating. The strength is strongly dependent on the grating etch depth. Having an etch of less than $70 \mathrm{~nm}$ would result in a weaker grating with a longer out-coupling length and thus a narrower beam. This would increase the sensitivity of the component.

\section{CONCLUSION}

A two-dimensional off-chip beam steerer on SOI has been presented. Steering ranges of about $15^{\circ}$ in the $\theta$-direction and $50^{\circ}$ in the $\phi$-direction were measured for a wavelength shift of $100 \mathrm{~nm}$. The maximum measured sensitivity of shift in the $\phi$-direction was $10.7^{\circ} / \mathrm{nm}$. The maximum wavelength resolution in 2-D was $2 \mathrm{~nm}$ and is limited by the beam width. This can be further improved by increasing the number of grating couplers and decreasing the strength of the out-coupling grating.

\section{REFERENCES}

[1] P. F. McManamon, P. J. Bos, M. J. Escuti, J. Heikenfeld, S. Serati, H. K. Xie, and E. A. Watson, "A review of phased array steering for narrow-band electrooptical systems," Proc. IEEE, vol. 97, no. 6, pp. 1078-1096, Jun. 2009.

[2] K. Van Acoleyen, W. Bogaerts, J. Jágerská, N. L. Thomas, R. Houdré, and R. Baets, "Off-chip beam steering with a one-dimensional optical phased array on silicon-on-insulator," Opt. Lett., vol. 34, no. 9, pp. 1477-1479, 2009.

[3] T. Chan, E. Myslivets, and J. E. Ford, "2-Dimensional beamsteering using dispersive deflectors and wavelength tuning," Opt. Express, vol. 16, no. 19, pp. 14617-14628, 2008.

[4] K. Van Acoleyen, W. Bogaerts, H. Rogier, and R. Baets, "Two-dimensional dispersive beam steerer fabricated on silicon-on-insulator," in Proc. Group IV Photonics, Beijing, China, 2010, pp. FC7-FC7.

[5] J. Y. Yang, X. Q. Jiang, M. H. Wang, and Y. L. Wang, "Two-dimensional wavelength demultiplexing employing multilevel arrayed waveguides," Opt. Express, vol. 12, no. 6, pp. 1084-1089, 2004.

[6] V. R. Supradeepa, C. B. Huang, D. E. Leaird, and A. M. Weiner, "Femtosecond pulse shaping in two dimensions: Towards higher complexity optical waveforms," Opt. Express, vol. 16, no. 16, pp. 11878-11887, 2008.

[7] The Silicon Photonics Platform ePIXfab, 2011.

[8] D. Vermeulen, S. Selvaraja, G. Verheyen, P. Lepage, W. Bogaerts, and G. Roelkens, "High-efficiency silicon-on-insulator fiber-to-Chip grating couplers using a silicon overlay," in Proc. Group IV Photonics, San Fransisco, CA, 2009, pp. FPd1-FPd1.

[9] N. L. Thomas, R. Houdré, M. V. Kotlyar, D. O’Brien, and T. E. Krauss, "Exploring light propagating in photonic crystals with Fourier optics," J. Opt. Soc. Amer. B, vol. 24, no. 12, pp. 2964-2971, 2007. 